\title{
The PixFEL project: development of advanced X-ray pixel detectors for application at future FEL facilities
}

\author{
G. Rizzo ${ }^{*, a, b}$, D. Comotti ${ }^{c, d}$, L. Fabris ${ }^{e, d}$, M. Grassi ${ }^{c, d}$, L. Lodola ${ }^{c, d}$, P. Malcovati ${ }^{c, d}$, \\ M. Manghisoni ${ }^{e, d}$, L. Ratti ${ }^{c, d}$, V. Re ${ }^{e, d}$, G. Traversi ${ }^{e, d}$, C. Vacchi ${ }^{c, d}$, G. Batignani ${ }^{a, b}$, \\ S. Bettarini ${ }^{a, b}$, G. Casarosa ${ }^{a, b}$, F. Forti ${ }^{a, b}$, F. Morsani ${ }^{b}$, A. Paladino ${ }^{a, b}$, \\ E. Paoloni $a, b$, G.-F. Dalla Betta $f, g$, L. Pancheri $f, g$, G. Verzellesi $h, g$, H. Xu $f, g$, R. \\ Mendicino $f, g$ and M.A. Benkechkache ${ }^{f, g}$ \\ ' Universita' di Pisa, Dip. di Fisica, I-56127, Italy \\ ${ }^{b}$ INFN, Sezione di Pisa, I-56127 Pisa, Italy \\ ' Universita' di Pavia, Dip. di Ingegneria Industriale e dell'Informazione, I-27100 Pavia, Italy \\ ${ }^{d}$ INFN, Sezione di Pavia, I-27100 Pavia, Italy \\ e Universita' di Bergamo, Dip. di Ingegneria e Scienze Applicate, I-24044 Dalmine (BG) Italy \\ ${ }^{f}$ Universita' di Trento, Dip. di Ingegneria Industriale, I-38123 Trento, Italy \\ g TIFPA, I-38123 Trento, Italy \\ ${ }^{h}$ Universita' di Modena e Reggio, I-41121 Modena, Italy \\ E-mail: giuliana.rizzo@pi.infn.it
}

ABSTRACT: The PixFEL project aims to develop an advanced X-ray camera for imaging suited for the demanding requirements of next generation free electron laser (FEL) facilities. New technologies can be deployed to boost the performance of imaging detectors as well as future pixel devices for tracking. In the first phase of the PixFEL project, approved by the INFN, the focus will be on the development of the microelectronic building blocks, carried out with a $65 \mathrm{~nm}$ CMOS technology, implementing a low noise analog front-end channel with high dynamic range and compression features, a low power ADC and high density memory. At the same time PixFEL will investigate and implement some of the enabling technologies to assembly a seamless large area X-ray camera composed by a matrix of multilayer four-side buttable tiles. A pixel matrix with active edge will be developed to minimize the dead area of the sensor layer. Vertical interconnection of two CMOS tiers will be explored to build a four-side buttable readout chip with small pixel pitch and all the on-board required functionalities. The ambitious target requirements of the new pixel device are: single photon resolution, 1 to $10^{4}$ photons @ $1 \mathrm{keV}$ to $10 \mathrm{keV}$ input dynamic range, 10-bit analog to digital conversion up to $5 \mathrm{MHz}, 1$ kevent in-pixel memory and $100 \mu \mathrm{m}$ pixel pitch. The long term goal of PixFEL will be the development of a versatile X-ray camera to be operated either in burst mode (European XFEL), or in continuous mode to cope with the high frame rates foreseen for the upgrade phase of the LCLS-II at SLAC.

KEYWORDS: X-ray detectors; Instrumentation for FEL; Solid state detectors.

${ }^{*}$ Corresponding author. 


\section{Contents}

1. Introduction 囵

2. FEL detectors requirements and challenges 2

3. PixFEL project overview

4. Active edge sensors

5. Front-end electronics \& multi-layer (3D) system architecture

6. Conclusions and outlook

\section{Introduction}

After the success of first generation X-ray free electron lasers (FEL) in the investigation of the microscopic structure of organic and inorganic materials in many fields (i.e. biology, chemistry, material science, atomic and molecular science), many reaserch centers worldwide have started studying, designing and building new FEL facilities with unique $\mathrm{X}$-ray beam properties, in term of peak brilliance, short pulse duration, high repetition rates [[1, [2, [3]. Table [1 shows the main features of some existing and planned FELs. Repetition rates differ widely between facilities, with the European XFEL producing bursts of high repetition rate $(4.5 \mathrm{MHz})$ packets separated by a $100 \mathrm{~ms}$ interval (burst operation mode) while the Stanford-based LCLS is operated at a constant pulse rate of $120 \mathrm{~Hz}$ (continuous operation mode). Several beam lines differing in photon energy and intensity are normally present to address different experimental requirements and techniques.

To fully exploit the potential of next generation FEL facilities, new X-ray imaging detectors need to be developed in order to match the very demanding requirements such as space and amplitude resolution, input dynamic range, frame rate and frame storage capability.

The PixFEL Collaboration has been recently approved by the INFN to carry on a research program for the development of a two-dimensional pixelated imaging camera for application at future FEL facilities, with the aim of advancing the state-of-art of imaging instrumentation by exploiting innovative solutions and new technologies also being explored in the HEP community for charged particle tracking pixel detectors.

The final goal is the development of a large area seamless X-ray imager for FEL applications based on hybrid multilayer tiles made of active-edge high resistivity pixel sensors bump-bonded to two-tiered 65-nm CMOS front-end ICs. The ICs are interconnected using through silicon vias (TSV) to build four-side buttable readout chips with a small pixel pitch and all the needed functionalities on board. 
Table 1. Main features of some existing and future FEL facilities.

\begin{tabular}{lccccc}
\hline Project & $\begin{array}{c}\text { Start of } \\
\text { operation }\end{array}$ & $\begin{array}{c}\text { Electron } \\
\text { energy } \\
{[\mathbf{G e V}]}\end{array}$ & $\begin{array}{c}\text { Photon } \\
\text { energy } \\
{[\mathbf{k e V}]}\end{array}$ & $\begin{array}{c}\text { Frame/Burst } \\
\text { rate } \\
{[\mathbf{H z}]}\end{array}$ & $\begin{array}{c}\text { Pulses } \\
\text { per } \\
\text { burst }\end{array}$ \\
\hline FLASH@DESY & 2005 & 1.25 & $0.03-0.3$ & 5 & $800 @ 1 \mu \mathrm{s}$ \\
LCLS@SLAC & 2009 & 14.5 & $0.3-10$ & 120 & 1 \\
Fermi@ELETTRA & 2010 & 2.4 & $0.01-0.06$ & 10 & 1 \\
SACLA@Spring-8 & 2011 & 8.5 & $4.5-15$ & 60 & 1 \\
Eu-XFEL@DESY & 2015 & 17.5 & $0.25-25$ & 10 & $2700 @ 220 \mathrm{~ns}$ \\
SwissFEL@PSI & 2016 & 5.8 & $1-12$ & 100 & $2 @ 50 \mathrm{~ns}$ \\
LCLS II@SLAC & $>2020$ & $4-14.5$ & $0.2-25$ & $120-10^{6}$ & 1 \\
\hline
\end{tabular}

In the initial three-year phase, the PixFEL project plans to realize a demonstrator to verify some of the proposed technologies, with the main focus on the development of the microelectronic building blocks, the exploration of the enabling technology for the multilayer tile, and the study of readout architectures for application at FEL facilities operated with high repetition rates in burst and continuous mode.

\section{FEL detectors requirements and challenges}

We will concentrate here on the measurement of the diffraction pattern generated by the scattering of coherent X-rays on a sample under test. A summary of the main requirements and the existing development projects follows, while a complete review can be found in [四].

FEL pulse structure: for many applications the camera should be able to digitize single shot images within the FEL repetition rate, which for the Eu-XFEL, is 220 ns. For burst operation mode, a series of frames (ideally the complete bunch train of 2700 pulses for Eu-XFEL) should be locally stored during the burst, to be read out later during the longer inter-train time. For continuous operation mode, frames must be read out continuously. For some applications at next generation FELs, the repetition rate may be as high as $1 \mathrm{MHz}$.

Energy range: ideally the same system should be able to cover the large photon energy range available at each facility, $0.25-25 \mathrm{keV}$ for Eu-XFEL.

Dynamic range: a dynamic range extending from single photon detection up to $10^{4}$ photons from a single pulse is required to properly reconstruct the diffraction pattern down to small angles.

Radiation tolerance: very large doses up to $10-1000$ MGy over 3 years are expected in some part of the detector; the sensor will be struck directly by the scattered photons receiving the full dose, while the readout electronics will be partly screened by the sensitive layer.

Space resolution and area coverage: the overall dimension of the sample and the minimum feature size drive the requirements on the angular resolution and coverage of the focal plane. Pixel sizes ranging from 700 to $20 \mu \mathrm{m}$ depending on the application and on the focal plane distance are required, while with a photon wavelength of about $0.1 \mathrm{~nm}$, a $60^{\circ}$ coverage is needed to detect substructures of the same dimension. This large angular coverage calls for a large area camera 


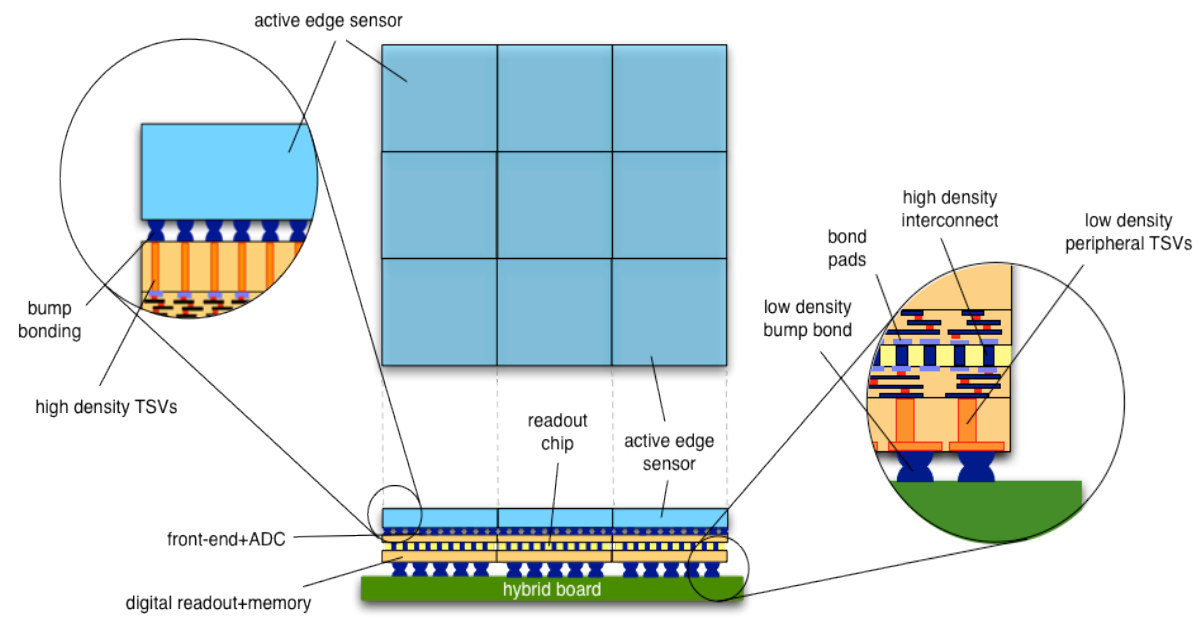

Figure 1. PixFEL matrix layout showing 9 four-side buttable modules, each consisting of a multilayer device resulting from the vertical interconnection of the sensor to a dual-tier front-end chip. The chip, in turn, is bump bonded to a hybrid board.

(about $400 \mathrm{~cm}^{2}$ ), typically composed by several adjacent tiles. The dead area must be minimized since the missing data can lead to ambiguities in the reconstruction of the sample image from the diffraction pattern.

Several instruments are under development to meet the growing demand from FEL machines. For instance for the Eu-XFEL: the AGIPD Detector [5], the LPD Detector [6], the DSSC Detector [Z]. All these projects are well advanced and plan to build a large area camera of about $20 \times 20$ $\mathrm{cm}^{2}$, based on detector modules with a dead area of about $10-15 \%$.

\section{PixFEL project overview}

The PixFEL project aims at improving the performance of X-ray imaging systems for FEL facilities by using cutting-edge microelectronic technological developments. The main points of performance improvement are: small pixel pitch while retaining high speed readout and local frame storage; large dynamic range with high resolution in-pixel analog-to-digital conversion; reduced module dead areas allowing large area tiling without missing data issues.

An overview of the proposed technologies is shown in figure 10 representing a PixFEL matrix:

Active edge sensor: fully depleted high resistivity silicon pixel sensor guaranteeing high quantum efficiency for hard X-ray conversion.

Dual-tier 65-nm CMOS readout ICs: essential to enable enough density to include the required amount of on-board intelligence in a target pitch of $100 \mu \mathrm{m}$; a dual-tier approach is envisaged to provide enough space for the in-pixel Analog to Digital (A/D) conversion and data storage.

Vertical interconnects: various types of 3D vertical interconnects are required to connect the sensor to the chip, the two tiers together, and the sensor-chip assembly to the readout hybrid. Bump-bonding, through silicon vias, and small pitch vertical integration techniques will be used. The combination of active edge sensor and vertical integration technology will allow the realization of four-side buttable modules with minimal dead area. 
Table 2. Main specifications for the PixFEL demonstrator tile.

\begin{tabular}{rlrl}
\hline Pitch: & $100 \times 100 \mu \mathrm{m}^{2}$ & Dead area: & $2 \%$ \\
Photon energy: & $1-10 \mathrm{keV}$ & Dynamic range: & $1-10^{4}$ \\
Memory: & $1 \mathrm{k}$ frame depth & A/D conversion: & $200 \mathrm{~ns}, 10 \mathrm{bit}$ ADC \\
\hline \multirow{2}{*}{ Readout: } & in burst mode, $4.5 \mathrm{MHz}$ frame rate, $1 \%$ duty cycle \\
& in continuous mode, $15 \mathrm{kHz}$ \\
\hline
\end{tabular}

The initial three-year phase of the PixFEL project will be concerned with: investigating the enabling technologies leading to a 4-side buttable elementary chip and to an almost zero deadarea detector, resulting from the assembly of these elementary blocks; developing and optimizing the microelectronic building blocks to be included in the front-end chip for a 2D X-ray camera; studying the architectures for high performance in-pixel storage and readout of the data.

Table 2 shows the main specifications for the PixFEL demonstrator tile.

For the full system, the goal is to obtain a $20 \times 20 \mathrm{~cm}^{2}$ detector composed of tiles of $2.56 \times$ $5.12 \mathrm{~cm}^{2}$, each assembled from a single high resistivity sensor and $32(4 \times 8)$ square readout ICs with $64 \times 64$ pixels.

\section{Active edge sensors}

In PixFEL, the minimization of the dead area of the sensors will be pursed by adopting active edge technology, first introduced at the Stanford Nanofabrication Facility as an extension of 3D detector technology [8]. To extend the sensitivity of the sensor up to a few micron from its physical edge, in active edge sensors the cut lines of each detector tile are obtained with etched and heavily doped deep trenches, realized by Deep Reactive Ion Etching (DRIE), to act as wall (ohmic) electrodes. Active edge planar sensors have been also developed at other processing facilities (SINTEF, VTT, FBK) and a review of recent results can be found in [ 9 ].

The PixFEL active edge sensors will be developed in collaboration with the Fondazione Bruno Kessler (Trento, Italy), taking advantage of the activities already going on for the pixel sensor for the ALICE and ATLAS upgrades with optimization for the specific X-ray imaging application for FEL instrumentation. A relatively thick sensor is needed (about $450 \mu \mathrm{m}$ ) to achieve a good detection efficiency for photons in the energy range from 1 to $10 \mathrm{keV}$. Furthermore, one peculiar aspect for applications at FEL is the impact of the so called plasma effect: in case a large number of photons (up to $10^{4}$ ) hits a single pixel, the resulting high charge density reduces the collection electric field and affects charge collection, point spread function and response time of the sensor. This effect can be mitigated by increasing the bias voltage [10] but early breakdown voltage then becomes a limiting factor for the detector operation. For this reason, specific TCAD device simulations have been performed to investigate the plasma effect and to optimize the edge termination in order to reach the best trade off between the minimization of the dead area and an acceptable breakdown voltage.

The simulated device is a p-on-n sensor with $100 \mu \mathrm{m}$ pitch pixel on a high-resistivity (n-) substrate, 300 or $450 \mu \mathrm{m}$ thick, with $\mathrm{n}+$ ohmic contact regions at the back-side and along the 

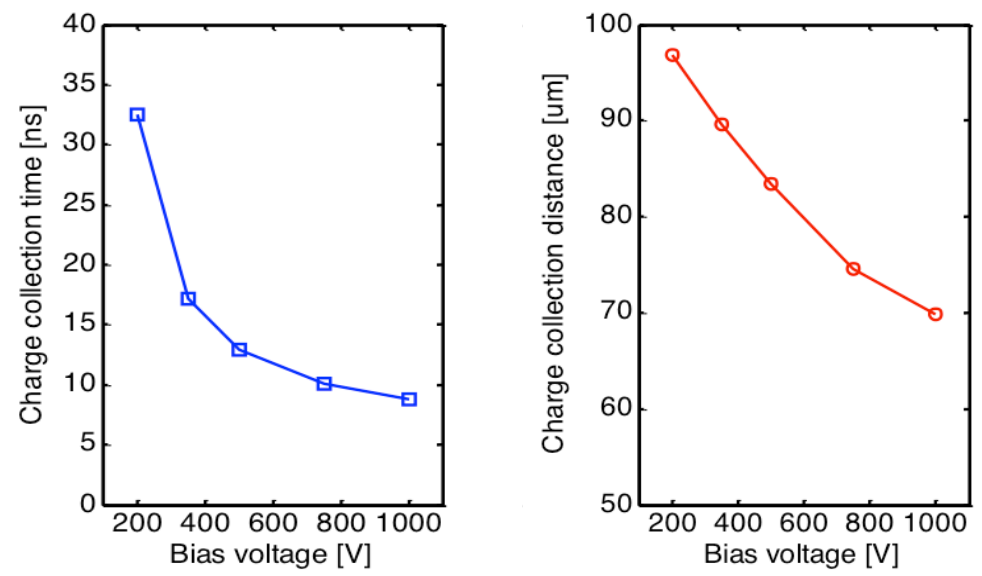

Figure 2. Simulated time and distance from the injection point, for $95 \%$ charge collection, as a function of bias voltage applied. Charge released in the active edge sensor was equivalent to $10^{4}$ photons at $12 \mathrm{keV}$.
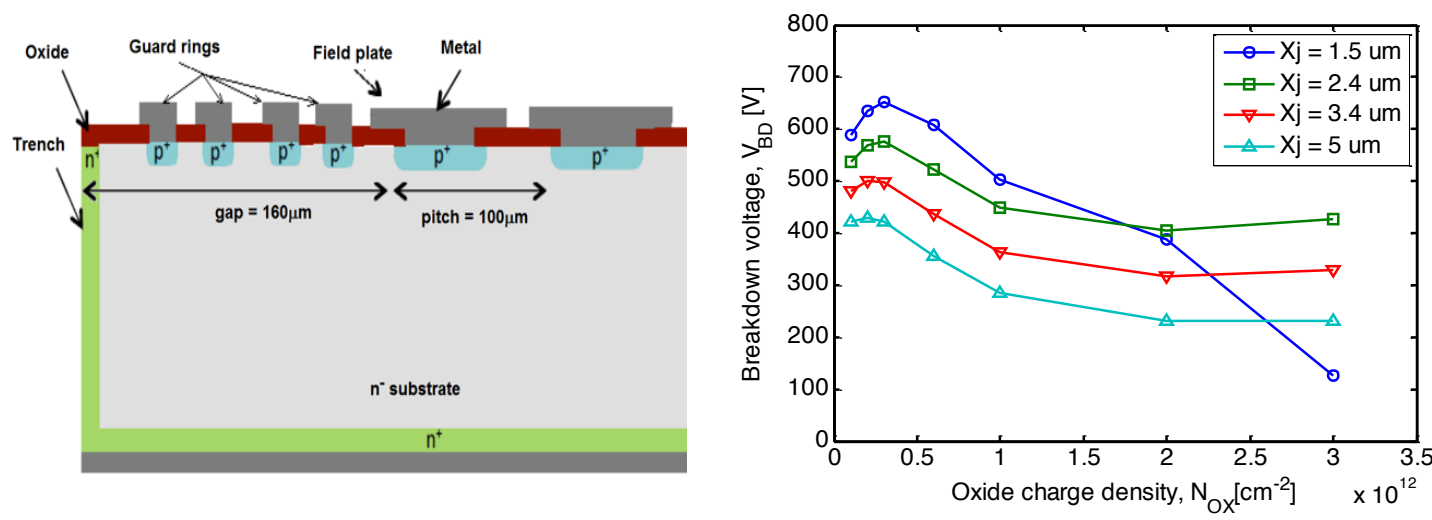

Figure 3. Schematic cross-section of the simulated active edge sensor (left). Breakdown voltage of the simulated device as a function of the surface oxide charge density and with various junction depths (right).

trench at the edge. To study the plasma effect 2D simulations with cylindrical coordinates have been carried out, using an optical model to mimic the energy released by a X-ray photon beam. The backside of the sensor was illuminated, varying the distance of the injection point with respect to the pixel center. Multiple narrow electrodes were also insterted within the $100 \mu \mathrm{m}$ pitch to evaluate the point spread function. Results shown in figure 2 are in good agreement with experimental data reported in [10], and indicate that collection times below $20 \mathrm{~ns}$ and collection distance below 90 $\mu \mathrm{m}$ can be achieved with a bias voltage of about $300 \mathrm{~V}$, even in the worst case condition, with up to $10^{4}$ photons of 1 and $12 \mathrm{keV}$.

To increase the breakdown voltage above these values, different edge sensor designs have been simulated, tuning the most sensitive parameters, like edge distance, number and shape of floating guard rings, field plate dimension, oxide thickness and junction depth. Best results have been optained with an oxide thickness of $300 \mathrm{~nm}$, a junction depth of $2.4 \mu \mathrm{m}$ and an edge termination with four guard rings with external field plates, resulting in a total gap of about $155 \mu \mathrm{m}$ between the last 


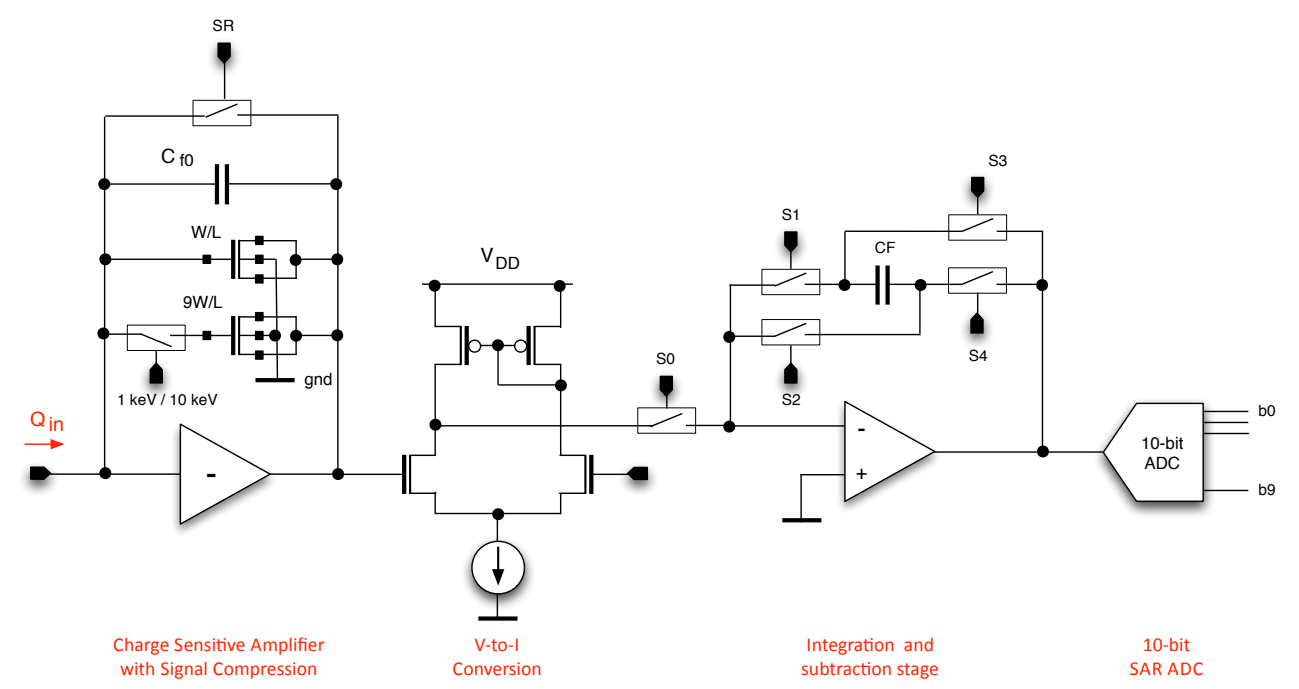

Figure 4. Schematic diagram of the full readout channel

pixel and the physical edge of the sensor. A schematic drawing of the proposed edge termination is shown in figure 3, together with the breakdown voltage results from TCAD simulations: values above $400 \mathrm{~V}$ have been obtained for the entire operation lifetime, even with an accumulated surface oxide charge density of $3 \times 10^{12} \mathrm{~cm}^{-2}$, which is expected after the exposure to 1 GGy of total ionizing dose.

With the small dead area achieved in this design, corresponding to only about $2 \%$ of the sensor tile, these active edge pixel sensors could represent a significant improvement with respect to other pixel devices presently under construction for FELs. First prototypes of the PixFEL active edge sensors will be submitted for fabrication at FBK by the end of 2014.

\section{Front-end electronics \& multi-layer (3D) system architecture}

Front-end Electronics. The development of the readout electronics, is carried out with a $65 \mathrm{~nm}$ CMOS technology to take full advantage of the synergy with similar activities starting in the HEP comunity for future detector development and at the same time to get increased in-pixel functionality and storage capability while reducing the pixel pitch.

The low noise front-end channel with wide input dynamic range is implementing a novel signal compression technique [11] based on the non-linear capacitance of a MOS used in the feedback network of the charge sensitive preamplifier (CSA), as shown in figure $\$$. The gain of the CSA is proportional to the inverse of the feed-back capacitance between the gate of the MOSFET and its source and drain, shorted together. When the signal collected is small (i.e. the gate-to-source voltage is much smaller than the threshold voltage) the capacitance of the MOSFET is very small, due to the sum of the gate-to-source and gate-to-drain overlap capacitances. For large signal, when the threshold voltage is exceeded, the MOS is operated in inversion mode, with source and drain channel linked, and the capacitance is dominated by the larger gate-to-channel capacitance. Such capacitance may increase even by a few order of magnitude, depending on the channel size. This 
non-linear feature of the MOS capacitor is exploited to dynamically change the sensitivity of the CSA with the input signal amplitude, then providing a dynamic signal compression.

With respect to other solutions proposed [四, the PixFEL channel has the advantage of being realized with standard CMOS technology and with a single amplifier with dynamically changing gain, instead of a parallel cofiguration with different switchable gains.

Digital data storage has been chosen for the PixFEL imager, since it provides a higher storage capability w.r.t. the analog approach [[]]. The A/D conversion will be in-pixel with a 10-bit successive approximation register (SAR) ADC. This solution provides an acceptable compromise between clock frequency and resolution; with 10 bits it provides both single photon resolution at small signal and quantization noise smaller than the Poisson noise for large signals, while retaining some margin for parameters dispersion and noise.

The full front-end channel, schematically shown in figure 4 , is described in details in [11]. It includes the CSA, a transconductor for voltage-to-current conversion, a time-variant filter realizing a trapezoidal weighting function and a 10-bit SAR ADC. The channel has been implemented in a first prototype chip, with an 8x8 pixel matrix, with $110 \mu \mathrm{m}$ pitch, submitted in Autumn 2014.

For operation with $50 \mathrm{~ns}$ integration time, results from post layout simulation give an Equivalent Noise Charge (ENC) of about $60 \mathrm{e}^{-}$, corresponding to a signal-to-noise ratio of about 4.6 for a single photon with an energy of $1 \mathrm{keV}$. The total power consumption of the channel is 350 $\mu \mathrm{W} /$ pixel, at $5 \mathrm{MHz}$ sampling rate.

System architecture. The PixFEL imaging camera will be a multi-layer device, based on 3D integration technology [12], [13], with a dual-tier readout chip with the first tier dedicated to the analog signal processing and digitization, and the second layer hosting the digital memories and the specific readout architecture.

In imaging detectors no sparsification is possible and the readout needs to deal with a large amount of data to be read out in a relatively short amount of time, depending on the time structure of the X-ray beam, which may change significantly from one FEL facility to the other.

A versatile design of the PixFEL architecture may include the possibility of selecting the readout method between a direct readout mode (for continuous operation FELs) and a store locally $\&$ read-out-later mode (for burst mode operation typical of the Eu-XFEL).

Burst Operation Mode. When the FEL is operated in burst mode with a very high repetition rate inside the train of photon pulses (e.g., at the Eu-XFEL), direct and complete data readout would require impractical bandwidths. In this case the common approach is to store as much information as possible in the pixel during the pulse train period and to send it out during the long inter-train period. Devices presently under contruction for the Eu-XFEL are able to store up to 650 frames, with a pixel pitch of about $250 \mu \mathrm{m}$ [Z]. To improve the storage capability in the small pixel pitch of $100 \mu \mathrm{m}$, the second layer of the PixFEL front-end chip, envisioned for the future evolution project, will be largely devoted to the integration of the digital memory, whose capacity may be close to 1 kword in a $65 \mathrm{~nm}$ CMOS process. The bandwidth needs for such readout architecture, operated in burst mode at the Eu-XFEL, are $0.6 \mathrm{~Gb} / \mathrm{s} / \mathrm{chip}$ and $20 \mathrm{~Gb} / \mathrm{s} / \mathrm{ladder}$, calculated for the present modularity of the PixFEL camera, that could be further optimized.

Continuous operation mode. For the relatively low repetition rates, of the order of 100 $\mathrm{Hz}$, foreseen in most of the FELs to be operated in continuous mode, direct readout of data of 
a megapixel imaging camera is within the reach of present technology. On the contrary the readout for devices to be operated at future FEL facilities with continuous repetition rates as high as $1 \mathrm{MHz}$ will be extremely challenging.

\section{Conclusions and outlook}

The PixFEL project is exploring the application of innovative technologies such as active edge sensors, $65 \mathrm{~nm}$ CMOS, through silicon vias, and vertical integration, to develop pixelated imaging camera for application at future FEL facilities. Beyond the initial demonstrator phase already approved by INFN, the long term ambitious plan is to develop a large area focal plane instrument with significantly improved performance beyond the state of the art especially for application at future machines with high repetition rates.

\section{Acknowledgments}

The research activity presented in this paper has been carried out in the framework of the PixFEL experiment funded by Istituto Nazionale di Fisica Nucleare (INFN).

\section{References}

[1] S. Baradaran et al., LCLS-II New Instruments Workshops Report. SLAC-R-993.

[2] Ultrafast Phenomena at the Nanoscale: Science opportunities at the SwissFEL X-ray Laser. PSI Bericht Nr. 09-10.

[3] Research possibilities at the European XFEL. www.xfel.eu/research/possibilities.

[4] H. Graafsma, Requirements for and development of 2 dimensional $X$-ray detectors for the European $X$-ray Free Electron Laser in Hamburg, 2009 JINST 4 P12011

[5] B. Henrich et al., The adaptive gain integrating pixel detector AGIPD, a detector for the European XFEL, Nucl. Instrum. Methods, A633 (2011) 511-514.

[6] M. Hart et al., Development of the LPD, a high dynamic range pixel detector for the European XFEL, in proceedings of 2012 IEEE Nuclear Science Symposium and Medical Imaging Conference (NSS/MIC) 534-537.

[7] M. Porro et al., Expected performance of the DEPFET sensor with signal compression: a large format $X$-ray imager with mega-frame readout capability for the European XFEL, Nucl. Instrum. Methods, A624(2010) 509-519.

[8] C.J. Kenney et al., Results from 3-D silicon sensors with wall electrodes: near-cell-edge sensitivity measurements as a preview of active-edge sensors, IEEE Trans. Nucl. Sci., 48 (2001) 2405-2410.

[9] G. F. Dalla Betta et al., Edgeless and slim-edge solutions for silicon pixel sensors, PoS (Vertex2013) 042

[10] J. Becker et al., Impact of plasma effects on the performance of silicon sensors at an X-ray FEL Nucl. Instrum. Methods, A615 (2010) 230-236.

[11] M. Manghisoni et al., Novel Active Signal Compression in Low-noise Analog Readout at Future FEL Facilities, in these Proceedings 
[12] R.S. Patti, 3D-IC for real chips and perspectives, TIPP2011, 2nd International Conference on Technology and Instrumentation for Particle Physics, June 9-14, 2011, Chicago (IL)

[13] D. Henry et al.,TSV last for hybrid pixel detectors, 2013 IEEE $63^{\text {rd }}$ Electronic Components and Technology Conference, pp. 568-575. 